\title{
A STUDY ON THE AUTOMATIC GENERATION PROGRAM OF PATTERN BASED FAULT DIAGNOSIS SYSTEM USING ANTLR
}

\author{
Jaehyun Kim and Yangsun Lee* \\ Dept. of Computer Engineering, Seokyeong University \\ 16-1 Jungneung-Dong, Sungbuk-Ku, Seoul 136-704, Korea \\ *yslee@skuniv.ac.kr
}

\begin{abstract}
IoT (Internet of Things) means the technology to connect to the Internet by embedding communication in various objects. Things connected to the IoT can have sensors built in to acquire data from external environments. As the IoT system becomes more common, many devices are being used in many ways. Since many equipment are connected to each other in these environments, it is important to diagnose a failure of the device because failure of one device can affect the other. However, since there are many different types of device, it is time-consuming and costly to create a fault detector for each device.

In this paper, we designed and implemented a program to automatically generate a fault diagnostic that determines whether the device failed based on sensor data collected from target device. The program consists of a pattern generator that produces a diagnostic pattern that indicates a specific fault for the target device and a fault diagnostic generator that generates a fault diagnostic that effectively diagnoses the fault through pattern analysis. In this paper, a fault diagnostic generator is implemented using ANTLR. Fault diagnostic detector generated through the implemented program can quickly diagnose faults in the target device.
\end{abstract}

Keywords - Internet of Things (IoT), Fault Diagnostic, Automatic Fault Diagnostic Generator, Pattern Generator, Fault Diagnostic Generator, ANTLR Parser Generating System

\section{Introduction}

IoT (Internet of Things) means the technology to connect to the Internet by embedding communication in various objects[1]. Things connected to the IoT can have sensors built in to acquire data from external environments. As the IoT system becomes more common, many devices are being used in many ways. Since many equipment are connected to each other in these environments, it is important to diagnose a failure of the device because failure of one device can affect the other. However, since there are many different types of device, it is time-consuming and costly to create a fault detector for each device[2-5].

In this paper, we designed and implemented a program to automatically generate a fault diagnostic that determines whether the device failed based on sensor data collected from target device. The structure of the automatic generation program of the diagnostic trouble machine consists of a pattern generator that produces a pattern of pseudo-code form that largely indicates a specific fault for the target device and a

Received: May 5, 2019

Reviewed: August 19, 2019

Accepted: August 28, 2019

* Corresponding Author 
fault diagnostic generator that generates a diagnostic that will practically diagnose the fault through pattern analysis.

The pattern generator consists of a data converter that processes the sensor data, a threshold calculator that calculates the threshold of the sensor when the target device is normal, a pattern determiner that extracts the sensor that is related to the fault, and a pattern filter that removes the non-fault sensor from the pattern. The fault diagnostic detector generation module is implemented using ANTLR[6]. Fault diagnostic generated through the implemented program can quickly diagnose faults in the target equipment. The pattern based fault diagnostic automatic generation program, implemented in this paper, can generate fault-diagnostic if sensor data of target equipment exist.

\section{RELATED STUDIES- ANTLR PARSER GENERATION SYSTEM}

ANTLR [6] is one of the open source, parser generation systems, and is drawing attention because it is convenient to use and has many advantages, including exception processing functions. Two components are used for a lexer and a parser for syntax analysis. The lexer is called a lexical analyzer or scanner, and reads the source program into a series of strings to create tokens. Token is used as a word to construct sentences in a minimum unit of grammatical significance, with five words: keyword, operator, separator, Identifier, and constant. The parser is called a syntax analyzer, and takes a token output by Lexer as an input to examine whether the source program is correct, generating a structure of sentences as a tree for the correct program. This tree is called a parse tree [7-11].

There are two ways for developers to implement the parser themselves or generate the parser through the parser generating system for parsing. Implementing a parser using a parser generating system is more efficient than implementing the parser directly because the programmers' grammar rules are defined and the parser generating system automatically generates the parser based on defined grammar. When a programmer defines the grammar rules of the text he wants to parse using a given expression, ANTLR, a type of parser generating system, produces the lexer and parser required for a syntax analysis. The lexer and parser can be used to perform the work of a given program, i.e., using a pars tree generated through the lexical analysis and the syntax analysis processes. For additional work with a parse tree, ANTLR offers two methods of tree searching: Listener and Visitor.

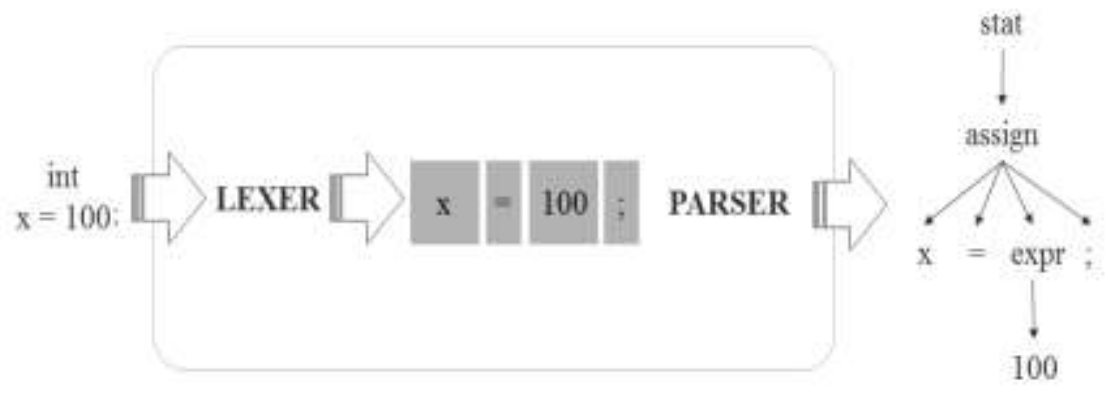

Fig. 1 Syntax Analysis by the ANTLR

\section{AUTOMATIC FAULT DIAGNOSIS GENERATION PROGRAM}

In this charter, we describe a method for automatically generating a fault diagnostic system to determine whether a fault has occurred in the device based on the sensor information of the target device and the data collected by the sensor. In principle, the sensor data, which is the basis for the fault diagnosis detector to determine if the target device fails, should contain both normal and abnormal values [12-14]. 
The automatic fault diagnostic generation program consists of a pattern generator module and a fault diagnostic generator module. Figure 2 shows the structure of the automatic fault diagnostic generation program.

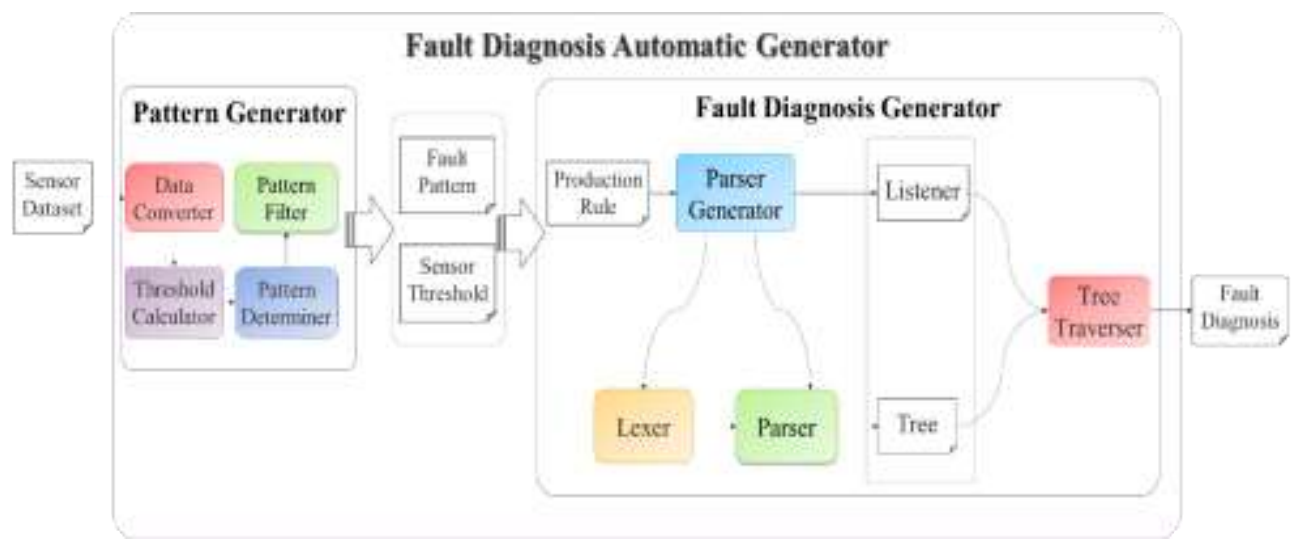

Fig. 2 Fault Diagnostic Automatic Generation Program

\subsection{PATTERN GENERATOR}

This section describes the pattern generator that receives sensor data as input and returns the fault diagnosis pattern and sensor threshold for later use in the fault diagnostic generator. The fault diagnostic pattern is defined using sensors that are deemed to be related to the fault, which is included in the sensor data. The sensor threshold defines the normal threshold of the sensor based on the value in the normal situation of the sensor. The pattern generator defines failure patterns and sensor threshold values through a total of four steps: data converter, threshold calculator, pattern detector, and pattern filter. Figure 3 shows the structure of the pattern generator.

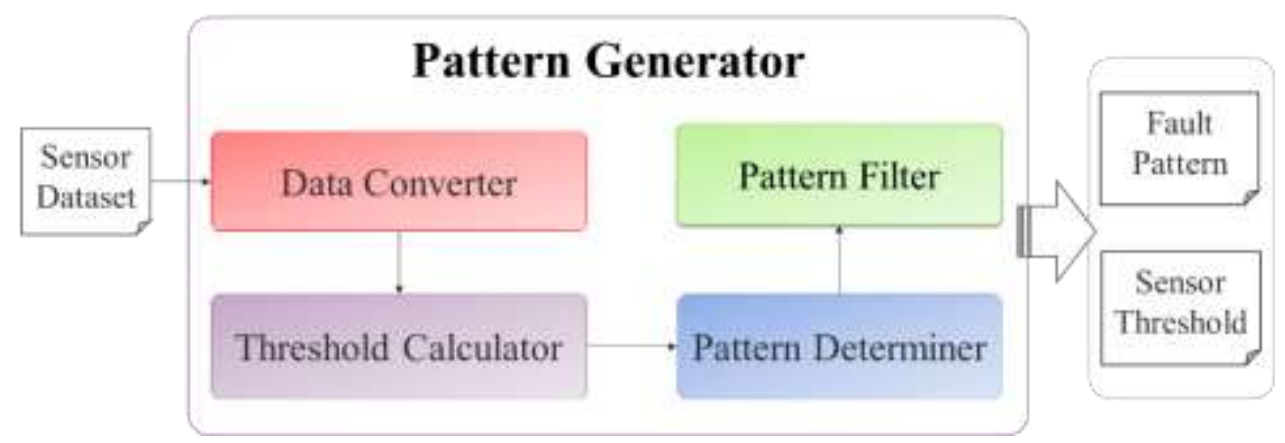

Fig. 3 Structure of the Pattern Generator

In this paper, we use the Advanced Diagnostics and Prognostics Testbed (ADAPT), which is an aerospace vehicle power system data provided by NASA [15]. This data is the data that has been injected with a fault at a specific point in time in normal circumstances and each experiment has a specific failure scenario and does not include a recovery situation for the fault.

3.1.1 DATA CONVERTER: In this section, the sensor data values of the data entered are differential and the sliding window is applied. Since the device is in the most stable condition when operating normally, changes in sensor values are relatively constant. However, if a particular fault occurs, the value of a sensor changes from normal situation due to the effect of the failure. In addition, the unit and size of sensor values vary as there 
are many different types of sensors, so there is a limit to selecting appropriate thresholds just for sensor data values for all sensors. Therefore, it is easy to establish thresholds by using differentials, or changes in values, rather than simple values. After differentiating, the sliding window is applied to clarify the range of normal values and to reduce fine errors. The size of the sliding window is 15 . This is an empirical constant. Figures 4, 5 and 6 show some of the graphs of the TE500 temperature sensor, the graph after differentiation, and the sliding window graph with 15 sizes respectively.

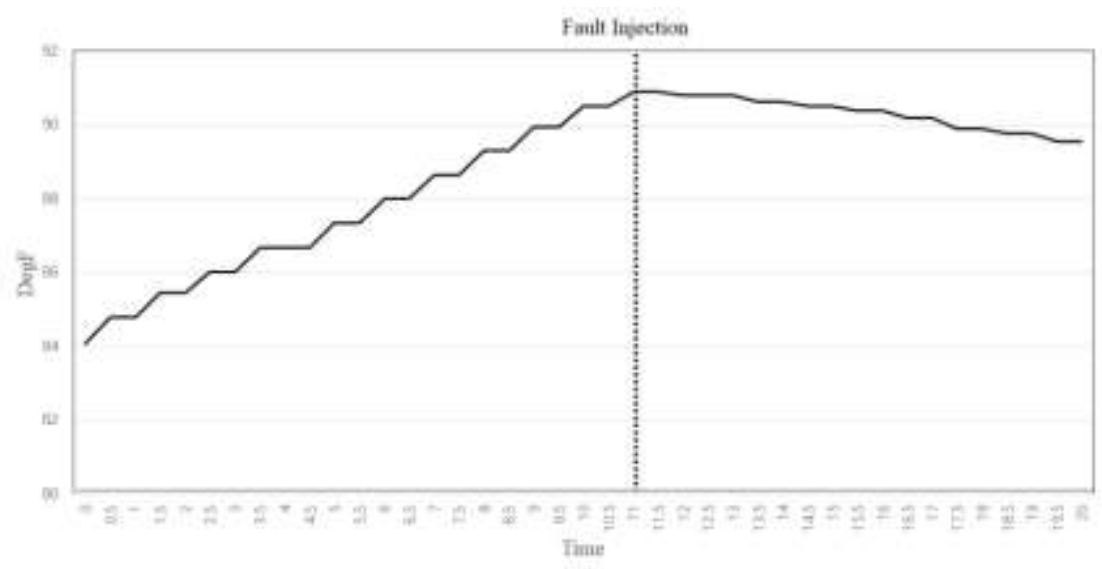

Fig. 4 Graph of the TE500 Temperature Sensor

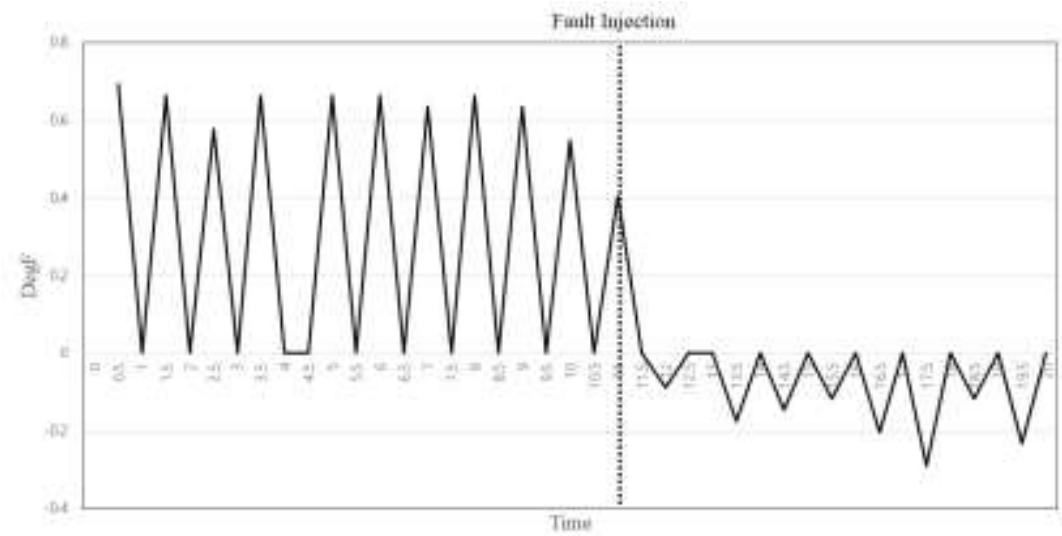

Fig. 5 Graph after differentiation of the TE500 Temperature Sensor

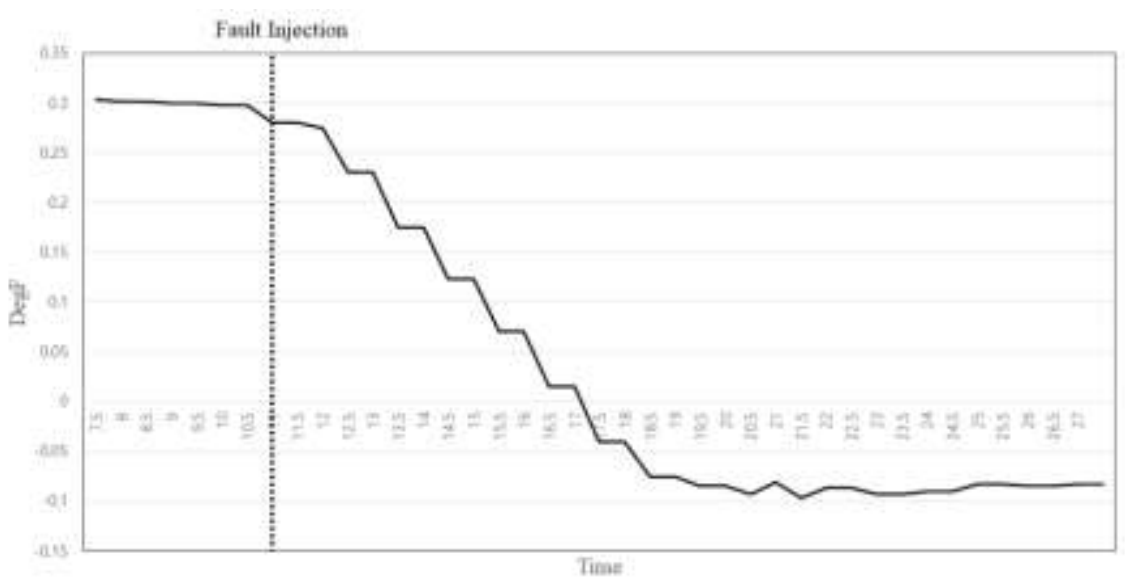

Fig. 6 Sliding Window Graph of the TE500 Temperature Sensor 
3.1.2 THRESHOLD CALCULATOR: In this section, the normal threshold for each sensor is determined using sensor data that has been completed up to the sliding window. Since it is a normal threshold, only data prior to the point of failure injection are used for the calculation of the threshold and this range is referred to as the normal range. Since the maximum and minimum values of the normal range are vulnerable to errors, the tolerance is set by setting.

In order to determine the threshold, the average of the maximum and minimum values is first obtained. Multiply the size of the mean to the maximum value by 1.2 (experience factor) to obtain the value of the allowable range. The addition of the values of the allowable range from the mean value shall be determined as the maximum value of the threshold and the subtraction as the minimum value of the threshold. Figure 7 shows the normal threshold graph of the TE500 temperature sensor.

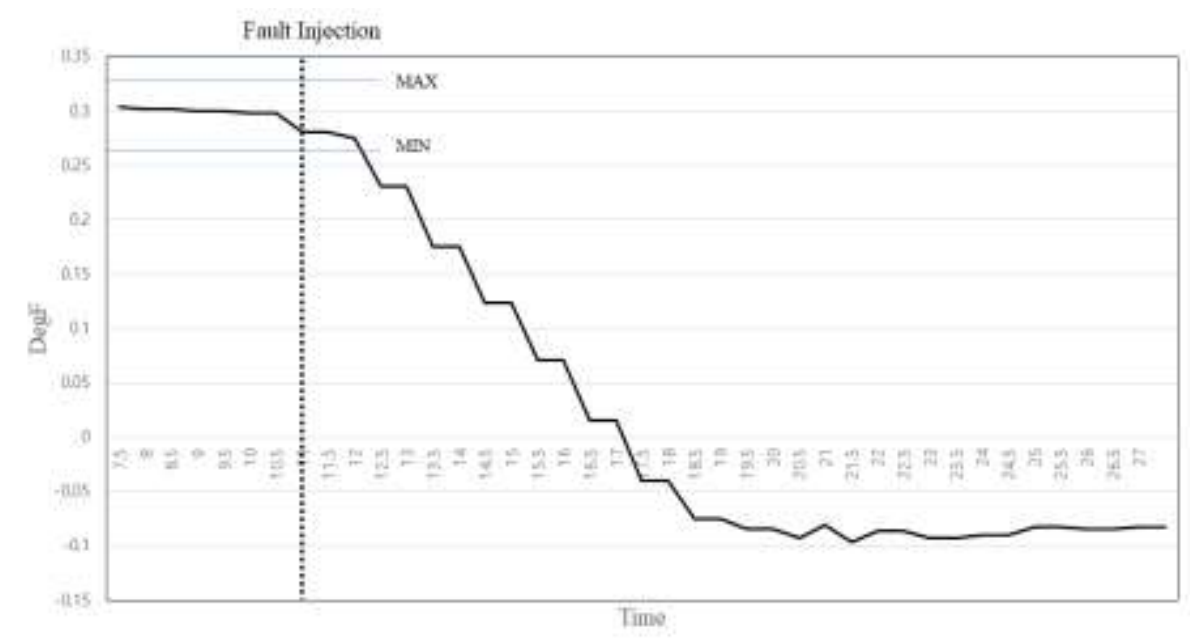

Fig. 7 Normal Threshold Graph of the TE500 Temperature Sensor

3.1.3. PATTERN DETERMINER: This section uses the thresholds of sensors set in the threshold calculator to add sensors associated with faults to the corresponding diagnostic pattern. Since the data used in this paper does not include the fault recovery situation, the failure condition is referred to as the failure condition from the point to the end of the failure injection of the sensor graph with the sliding window applied. In this paper, it is hard to say that the change in value is closely related to the fault after a certain period of time after the injection of the fault because the change in sensor value is used. Thus, only changes in sensor values that are closely related to the fault are made to establish the effective time for the fault test to be added to the fault diagnostic pattern. The effective time of the fault check is from the time of the failure injection to the point where the time of the normal range has elapsed. Figure 8 shows the validity of the failure test of the TE500 temperature sensor. 


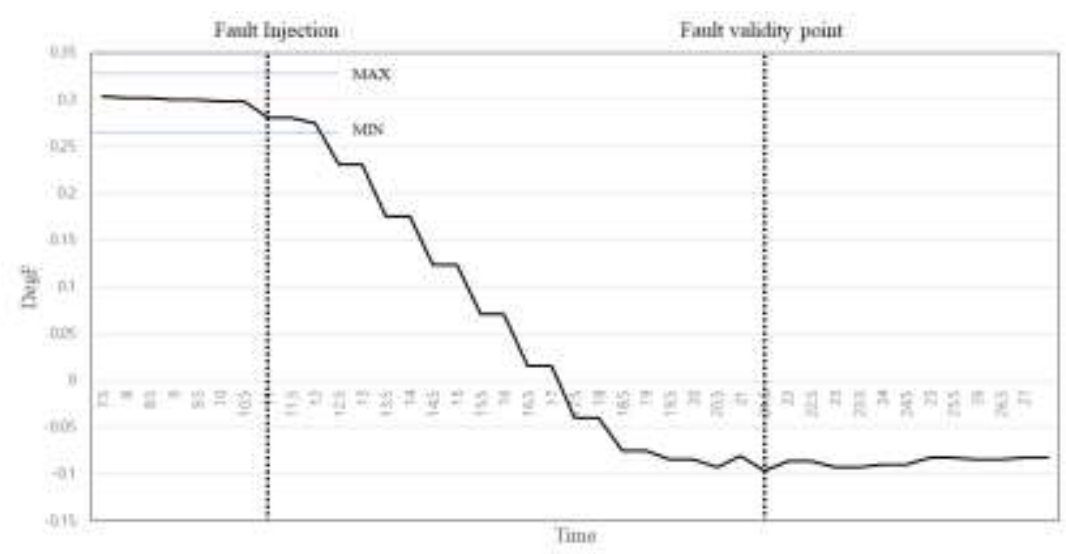

Fig. 8 Validity of the Failure Test of the TE500 Temperature Sensor

Compare the values and thresholds within the validity of each sensor failure to add the sensor to the pattern with DeltaHigh if it is greater than the maximum value of the threshold and DeltaLow if it is less than the minimum value. Afterwards, check if any of the sensors included in Delta High and DeltaLow are duplicated and if any, exclude them from the pattern.

3.1.4. PATTERN FILTER: This section removes the normal data low sensor from the pattern as a result of the previous section. Data for the same fault can also be added as a diagnostic pattern for the fault even though there is no sufficient amount of data in the normal range depending on the type and characteristics of the sensor and therefore the threshold of the normal situation has been incorrectly measured and not affected by the fault. This is referred to as normal low data sensor. The normal low data sensor is determined by two conditions.

The first condition is that "Values smaller than the change threshold of the corresponding sensor exist as long as the normal time width after the failure effective time." This condition is because the value of the normal range exists after the effective time of the failure by the normal time width, which can be considered to be the starting point of the overall data pattern. Figure 9 shows a graph of a sensor meeting the first condition.

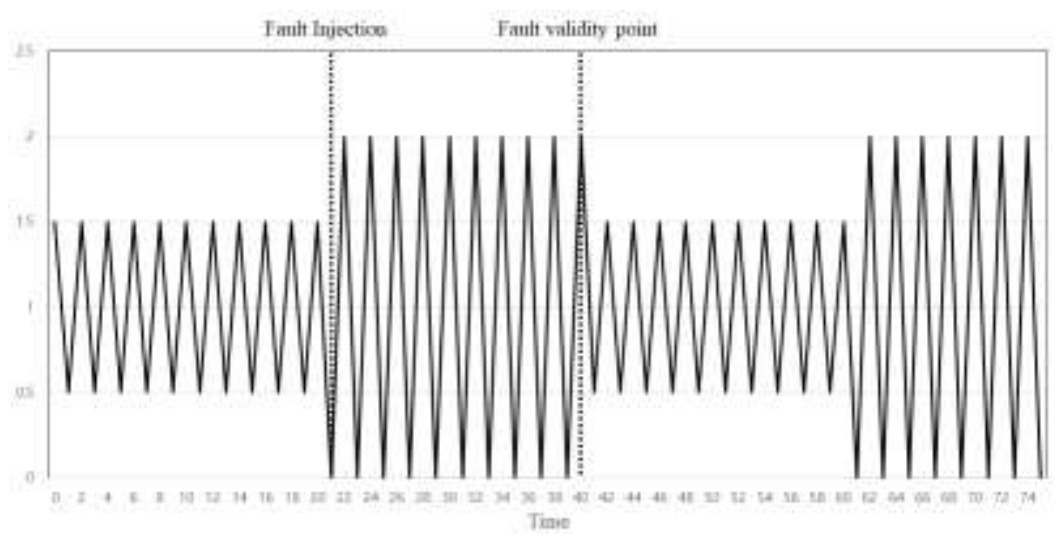

Fig. 9 Sensor Graph meeting the First Condition

The second condition is that "Values greater than the maximum absolute value of the failure effective time range are after the failure effective time." If device is operated normally, changes in sensor values will be maintained at a constant rate, and if a fault is affected, sensor values will change greatly. However, if there is a value greater than the maximum absolute value present within the failure effective time 
range after the failure effective time, this is judged to be a simple repetition of the pattern independent of the change due to the effects of the failure. Figure 10 shows a graph of a sensor meeting the second condition.

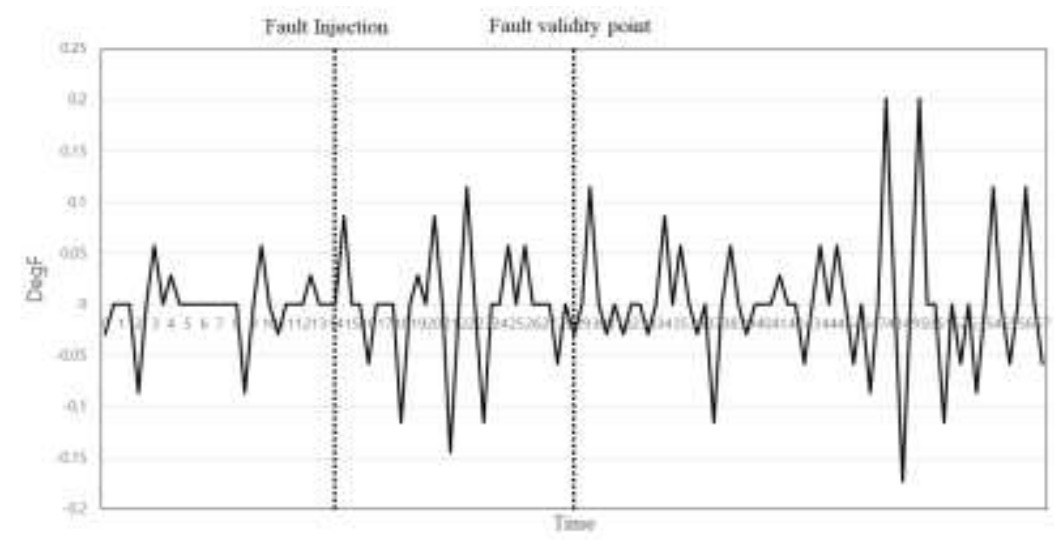

Fig. 10 Sensor Graph meeting the Second Condition

Once the data set entered into the pattern generator has gone through the pattern filter phase, the diagnostic pattern of the fault and the normal threshold of each sensor are generated. Table I shows the fault diagnostic patterns generated for faults in INV1, EY260, and CB262. In the table, DH represents DeltaHigh and DL represents DeltaLow.

Table I. Fault Diagnostic Patterns

\begin{tabular}{|c|l|}
\hline $\begin{array}{c}\text { Pattern } \\
\text { Number }\end{array}$ & \multicolumn{1}{|c|}{ Fault Diagnosis Pattern } \\
\hline Pattern1 & $\begin{array}{l}\text { DH(E135) \&\& DH(E140) \&\& DL(E167) \&\& DL(TE500) \&\& } \\
\cdots \text { DL(IT340) = INV1FailedOff }\end{array}$ \\
\hline Pattern2 & $\begin{array}{l}\text { DH(E181) \&\& DH(E335) \&\& DL(ST265) \&\& DL(IT161) \&\& } \cdots \\
\text { DL(TE510) = CB262FailedOpen }\end{array}$ \\
\hline Pattern3 & $\begin{array}{l}\text { DH(E235) \&\& DH(E242) \&\& DL(LT505) \&\& DL(TE507) \&\& } \\
\cdots \text { DL(IT240) = EY260Stuck }\end{array}$ \\
\hline
\end{tabular}

\subsection{FAULT DIAGNOSIS GENERATOR}

The fault diagnosis generator receives fault diagnostic patterns and thresholds generated from the pattern generator. At this stage, a fault diagnostic is generated to identify the failure of the target device, which is done by pattern analysis based on the threshold. In this paper, the work of changing pattern to tree structure is carried out as a way to do pattern analysis. Lexical analysis and syntax analysis should be performed as a prior task to changing patterns into tree structure [15-16].

To analyze whether a fault diagnostic pattern is made up of defined tokens and whether it is configured with the correct syntax structure, a lexer and a parser should be implemented, respectively, but these components can be generated from the parser generating system. In this chapter, a lexer and the parser is generated using the parser generating system, ANTLR. Figure 11 shows the structure of the fault diagnostic generator. 


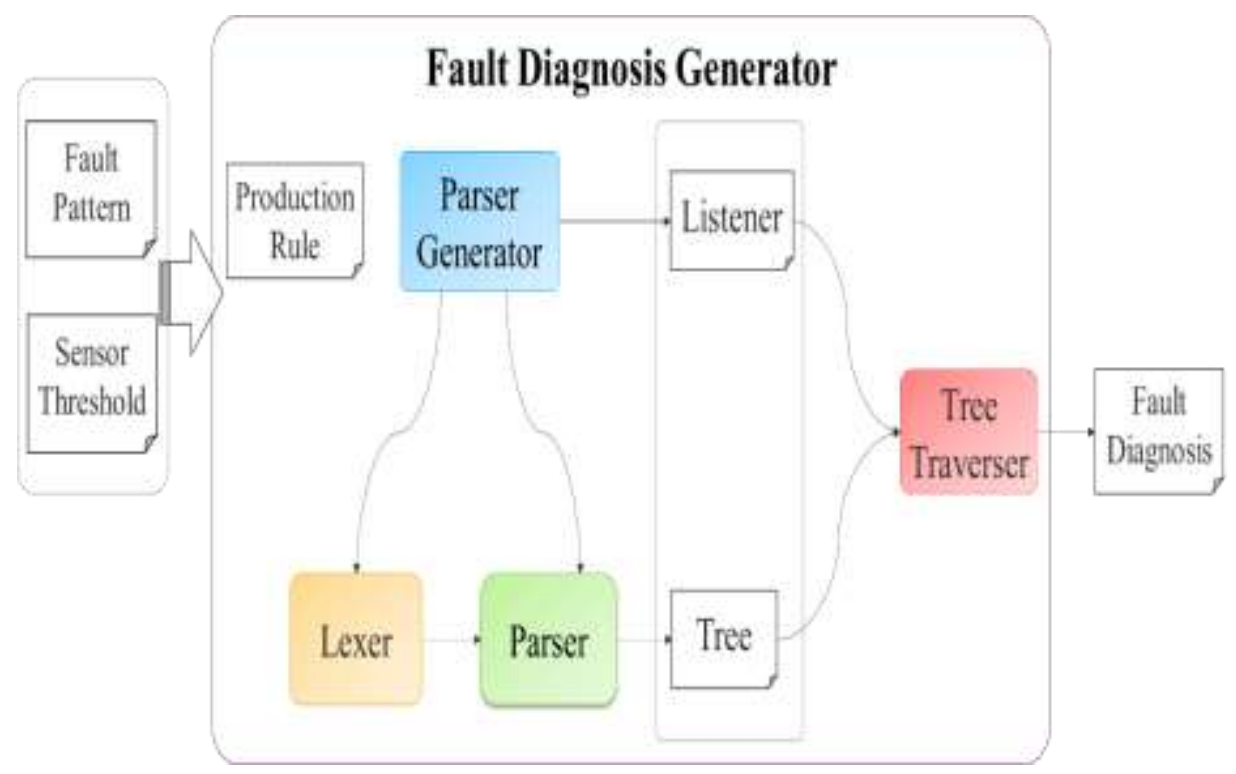

Fig. 11 Structure of the Fault Diagnostic Generator

3.2.1 PRODUCTION RULES: The production rule refers to the whole of token representation and grammar rules. Token expressions define the tokens present in the fault diagnostic pattern, and grammar rules define the syntax structure of the tokens, which are referred to as the Lexer Rule and the Parser Rule.

ANTLR requires a generation rule to generate a parser for a fault diagnostic pattern. Therefore, we have defined the Lexer rule and the Parser rule of the fault diagnostic pattern. Table II shows the production rules of the fault diagnostic patterns.

Table II. Production Rules of the Fault Diagnostic Patterns

\begin{tabular}{|l|}
\hline \multicolumn{1}{|c|}{ Pattern_Grammar.g4 } \\
\hline grammar patterngrammar; \\
diagnosis : (pattern)+; \\
pattern : test '==' STATE; \\
test : (andtest I ortest)+; \\
andtest : function ('\&\&' function)+; \\
ortest : function ('Il' function)+; \\
function : FUNCTIONLIST '(' SENSORNAME ')'; \\
STATE : 'normal' I 'INV1FailedOff' | 'EY260Stuck' \\
I 'CB262FailedOpen'; \\
FUNCTIONLIST : 'deltalow' I 'deltahigh'; \\
SENSORNAME : ('a'..'z'|'A' ..'Z') ('a'..'z'|'A' ..'Z'|'0'..' '9')*; \\
\hline
\end{tabular}

3.2.2. PARSE TREE: Parse tree means that the parser represents the structure of the sentence in the form of a tree for the correct sentence. To change the fault diagnostic pattern to a pars tree, enter the production rule defined earlier in the ANTLR to generate the Lexer and Parser. Table III shows the Parser code for the fault diagnostic pattern. 
Table III. Parser code for the Fault Diagnostic Pattern

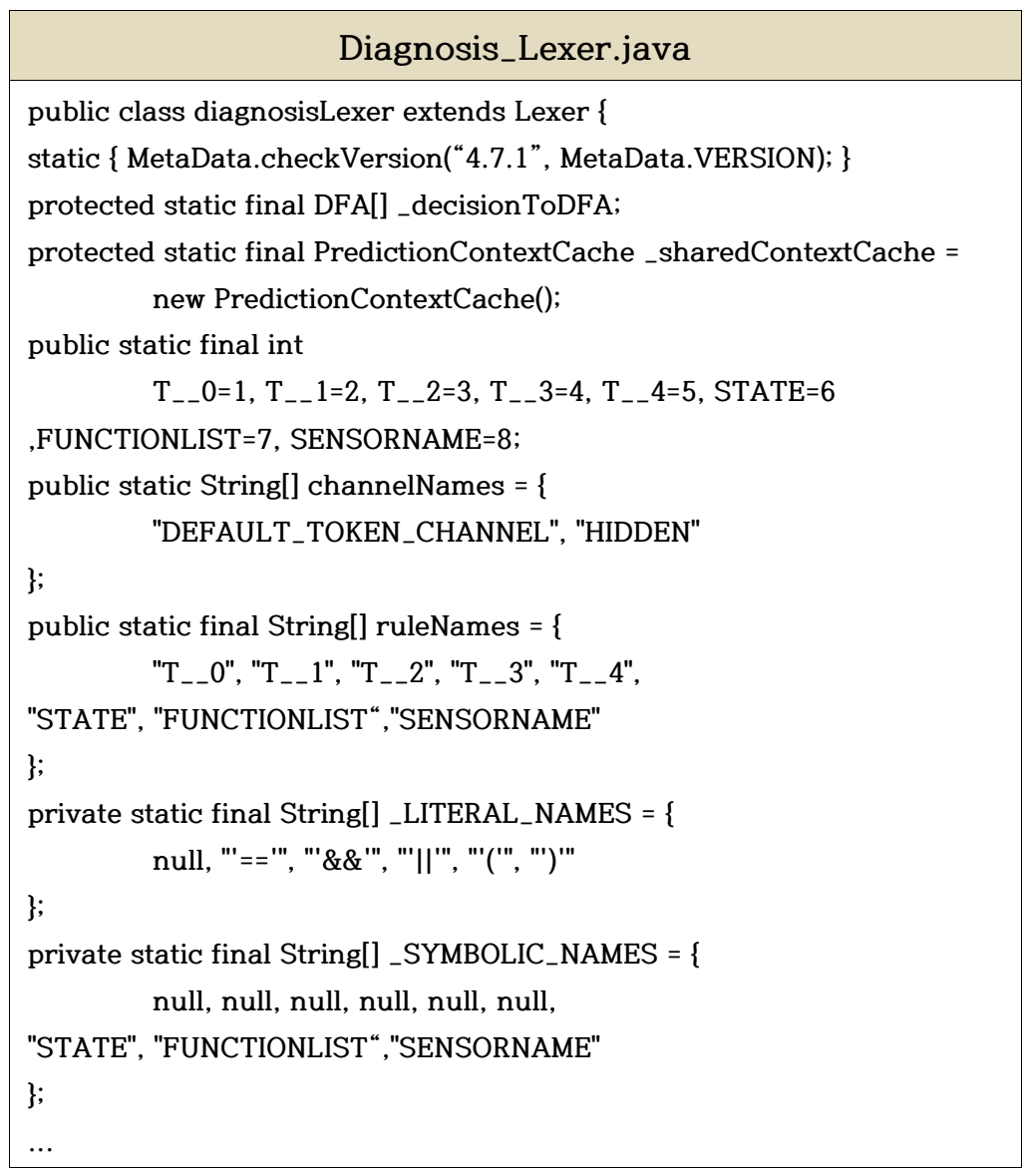

Entering a pattern into the Lexer results in token information, and typing the token information into the Parser produces a pars tree that has structured the pattern. Figure 12 shows part of the pars tree generated through the parser.

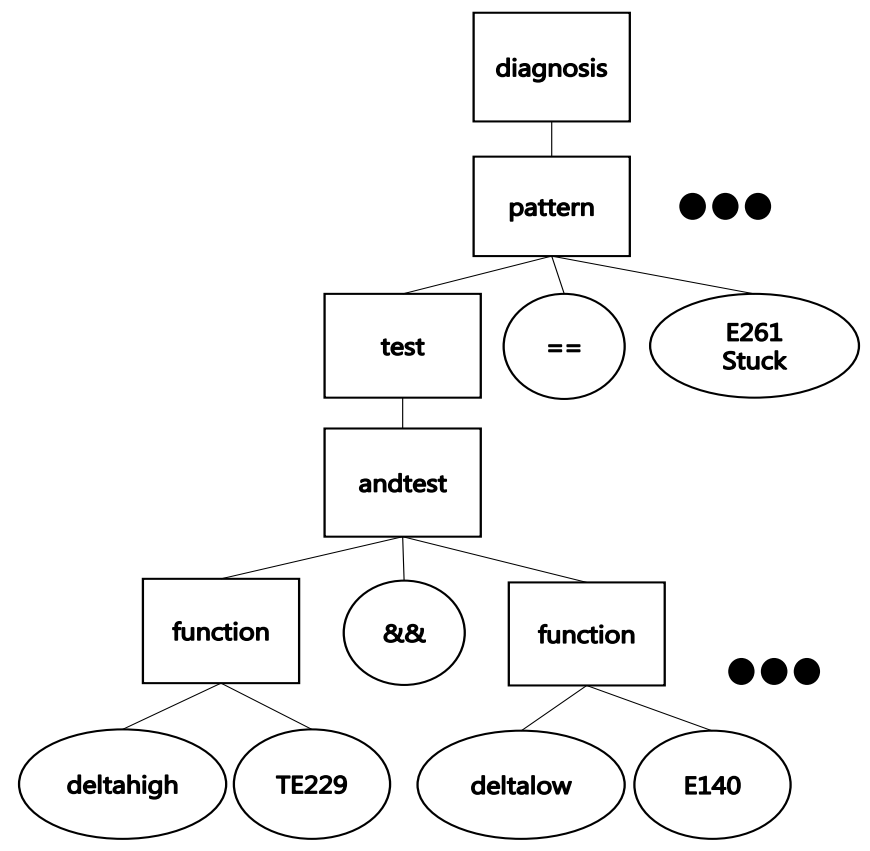

Fig. 12 Parse Tree generated through the Parser 
3.2.3. PROGRAM COMPLEXITY SECTION LEARNING: To analyze the pars tree, a method of visiting each node that exists in the tree is required. The Listener method was used to perform a tree analysis. The Listener consists of functions that approach terminal and nonterminal nodes. When searching the tree through the ParseTreeWalker class, the Listener approaches sequentially from the parent node to the child node. However, we used a bottom-up analysis method that visits from lower to higher nodes. Therefore, only a fraction of the functions within the Listener are implemented. Table IV shows the operation order of the overridden function.

Table IV. Operation Order of the Overridden Function

\begin{tabular}{|l|l|}
\multicolumn{2}{|c|}{ Diagnosis_Listener } \\
\hline & (7) exitFunction(FunctionContext) \\
(1) visitTerminal(TerminalNode) & (8) exitAndtest(AndtestContext) \\
(2) visitTerminal(TerminalNode) & (9) exitTest(TestContext) \\
(3) exitFunction(FunctionContext) & (10) visitTerminal(TerminalNode) \\
(4) visitTerminal(TerminalNode) & $(11)$ visitTerminal(TerminalNode) \\
(5) visitTerminal(TerminalNode) & $(12)$ exitPattern(PatternContext) \\
(6) visitTerminal(TerminalNode) & $\ldots$ \\
\hline
\end{tabular}

There is a terminal node called DeltaHigh, DeltaLow at the bottom of the tree. The DeltaHigh is a node that represents a high change, and when approached on a tree circuit, it goes to a mapping function called the DeltaHigh by visiting a node that represents the name of the corresponding sensor. These mapping functions determine the condition of the sensor based on the threshold value of each sensor data generated from the pattern generator. Figure 13 below shows the code of the implemented the DeltaHigh function.

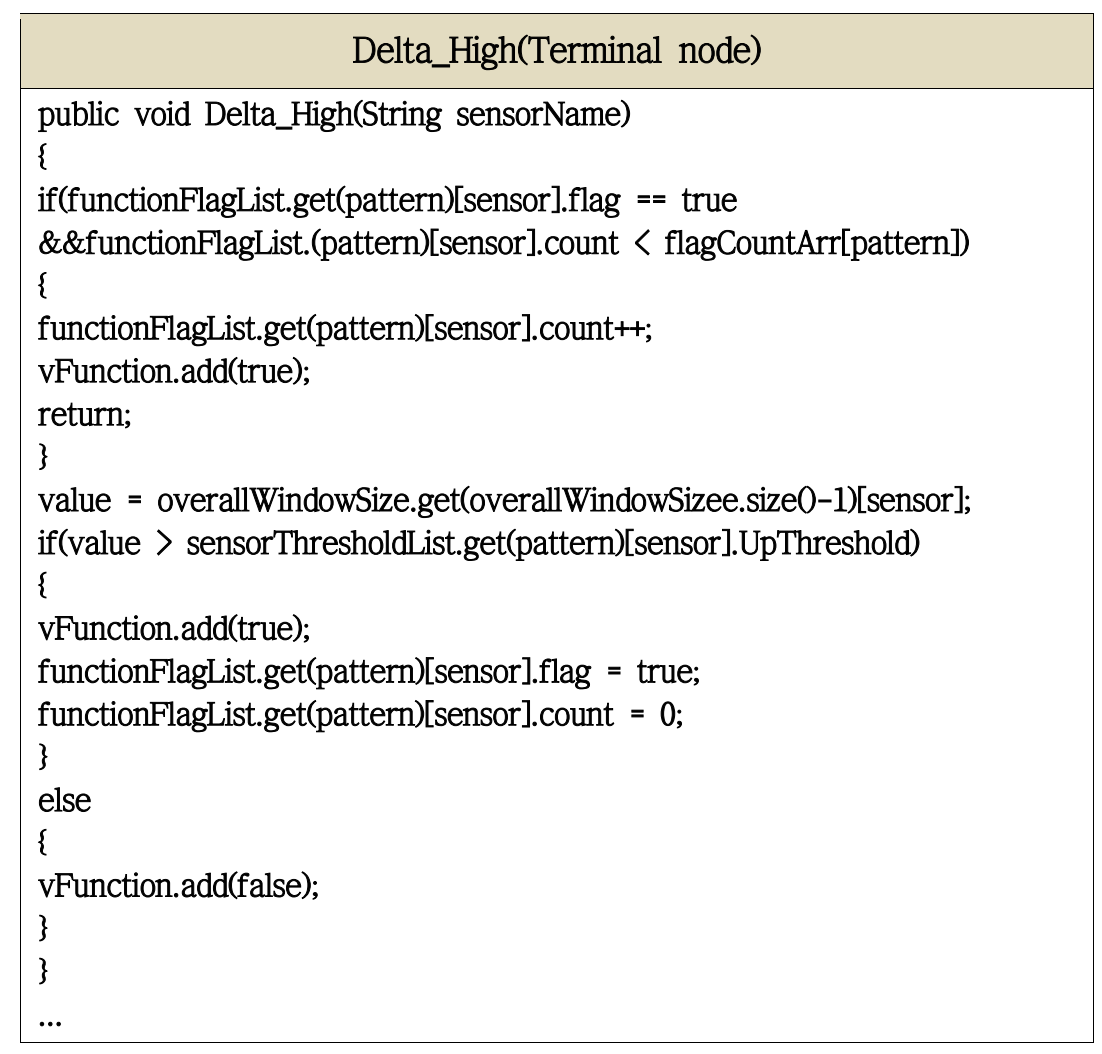

Fig. 13 Delta_High Function 
The mapping function allows the corresponding sensor to pass the status 'true' to the function node, the non-verbal node that exists at the top. This paper diagnoses faults that conform to the pattern in this way.

\section{EXPERIMENTAL RESULTS AND ANALYSIS}

In this chapter, the experiment was carried out with a fault diagnostic detector generated from the automatic fault diagnostic detector generation program designed by this research team. The generated fault diagnostic detector shows the experimental results of diagnosing the fault at a fast time. Table $\mathrm{V}$ shows the actual failure occurrence time of the ADAPT dataset.

Table V. Actual Failure Occurrence Time of the ADAPT Dataset

\begin{tabular}{|c|c|c|c|}
\hline \multirow{2}{*}{ Experiment } & \multicolumn{3}{|c|}{ Fault Description } \\
\cline { 2 - 4 } & Component & Mode & Time(s) \\
\hline 304 & EY260 & Stuck & 28 \\
\hline 306 & CB262 & FailedOpen & 47 \\
\hline 310 & INV1 & FailedOff & 29 \\
\hline
\end{tabular}

Table VI shows the fault occurrence time diagnosed by the fault diagnosis detector. The generated fault diagnosis detector diagnoses the fault at a time when the actual fault occurrence time does not greatly differ from the actual fault occurrence time.

Table VI. Fault Occurrence Time diagnosed by the Fault Diagnosis Detector

\begin{tabular}{|c|c|c|c|}
\hline \multirow{2}{*}{ Experiment } & \multicolumn{3}{|c|}{ Fault Description } \\
\cline { 2 - 4 } & Component & Mode & Time(s) \\
\hline 304 & EY260 & Stuck & 31 \\
\hline 306 & CB262 & FailedOpen & 56 \\
\hline 310 & INV1 & FailedOff & 31 \\
\hline
\end{tabular}

\section{CONCLUSIONS AND FURTHER RESEARCHES}

As the IoT system becomes popular, various devices are connected to the Internet and used. In this environment, as many devices are connected together, it is important to diagnose the failure of one device because failure of one device can affect other devices. However, it is both time-consuming and costly to build fault-diagnosis setector for as many different devices as possible.

In this paper, in order to solve this problem, the automatic generation program of the fault diagnosis system implemented by the research team receives the data set including both the sensor values when the target device is normal and the sensors when the target device is abnormal, and generates a meaningful pattern indicating a specific fault situation, and analyze the patterns and generate a fault diagnostics detector. Through the generated fault diagnosis detector, it is possible to diagnose faults by inputting sensor data existing in the target device in real time.

Future studies will verify whether the fault diagnostic detector generated by the sensor data sets of other target devices can provide an accurate fault diagnosis. 


\section{ACKNOWLEDGEMENTS}

This research was supported by Seokyeong University in 2019.

\section{REFERENCES}

[1] C. Michael, L. Markus, R. Roger, "The Internet of Things," McKinsey Quarterly, (2014).

[2] Y. Son, J. Jung, Y. Lee, "An Adaptive Offloading Method for an IoT-Cloud Converged Virtual Machine System Using a Hybrid Deep Neural Network," Sustainability, Vol.10, No.11, (2018), pp. 1-15.

[3] Y. Son, Y. Lee, "Offloading Method for Efficient Use of Local Computational Resources in Mobile Location-Based Services Using Clouds," Mobile Information Systems, Netherlands Hindawi Publishing Corp., Vol.2017, (2017), pp. 1-9.

[4] K. Kumar, "A Survey of Computation Offloading for Mobile Systems," Mobile Networks and Applications, Vol. 18, No. 1, (2013), pp. 129-140.

[5] K. Yang, S. Ou, and H.H. Chen, "On Effective Offloading Services for Resource-Constrained Mobile Devices Running Heavier Mobile Internet Applications," IEEE Comm. Magazine, Vol. 46, No. 1, (2008), pp. 56-63.

[6] T. Parr, The Definitive ANTLR4 Reference: Building Domain-Specific languages, The Pragmatic Bookshelf, (2013).

[7] K. Cooper, L. Torczon, Engineering a Compiler, Morgan Kaufmann, (2011).

[8] H. S. Mohan, Compiler Design, Morgan \& Claypool, (2014).

[9] T. Parr, Language Implementation Patterns, the Pragmatic Bookshelf, (2009).

[10] A. V. Aho, M. S. Lam, R. Sethi, and J. D. Ullman, Compilers: Principles, Techniques, and Tools, Addison-Wesley, Second ed., (2006).

[11] J. P. Tremblay, P. Sorenson, The Theory and Practice of Compiler Writing, McGraw-Hill, (1985)

[12] M. S. Mouchaweh, Fault Diagnosis of Hybrid Dynamic and Complex Systems, Springer, (2019).

[13] S. Narasimhan, G. Biswas, "Model-Based Diagnosis of Hybrid Systems," IEEE Transactions on Systems Man and Cybernetics, Vol. 37, No. 3, (2007), pp. 348-361.

[14] D. Marr, E. Hildreth, "Theory of Edge Detection," Proceedings of the Royal Society of London. Series B, Biological Sciences, Vol. 207, No. 1167, (1980), pp. 187-217.

[15] S. Poll, Advanced Diagnostics and Prognostics Testbed (ADAPT), Dashlink, (2016),

[16] Y. Lee, Y. Son, "A Study on the Smart Virtual Machine for Executing Virtual Machine Codes on Smart Platforms," International Journal of Smart Home, SERSC, Vol. 6, No. 4, (2012), pp. 93-105.

[17] Y. Lee, Y. Son, "A Study on the Smart Virtual Machine for Smart Devices," Information -an International Interdisciplinary Journal, Vol. 16, No. 2, International Information Institute, (2013), Japan, pp.1465-1472. 